kidney. He accordingly tested the kidney for amyloid degeneration; and on the application of Liquor Iodi it gave that peculiar staining, the nahogany tinge, characteristic of amyloid disease. The spleen was dense and very hard, had a fleshy feel, but was not enlarged. The liver was very much thickened in its whole structure, very hard, dense, and, if he might borrow a word from Professor Smith, carnified in its structure, like the spleen. The liver weighed 45 ounces.-February $25,1871$.

\title{
PROCEEDINGS OF THE DUBLIN OBSTETRICAL SOCIETY.
}

\section{THIRTY-SECOND SESSION.}

\section{Dr. KIDD, President.}

Dr. Atthinl read a paper on Some Forms of Menorrhagia.-He observed that there was not any symptom of uterine distase of more frequent occurrence, of greater gravity, or which causes more alarm to the patient than menorrhagia. It depends for its origin on such a variety of causes, local and constitutional, that the exercise of great caution is necessary as to diagnosis and to treatment. In the present communication, Dr. Atthill confined himself to the consideration of the treatment most suitable to cases of menorrhagia when occurring in connexion with, or dependent upon, sub-involution of the uterus, on granular ulceration of the cervix, or on an unhealthy condition of the mucous membrane being the body of the uterns.

Dr. Atthill regards defective involution of the uterus after labour or abortion, as a prominent cause of excessive menstruation. When subinvolution exists, an undue amount of blood is present in the organ, and the relaxed condition of the uterine tissue favours its exudation, therefore, when the periodic determination of blood to the uterus takes place, as occurs at each menstrual period, the moderate flow which should relieve that congestion becomes a profuse discharge, and often an exhausting drain. The mischief does not end here, for the abnormal state of the uterus in cases of sub-involution predisposes to granular ulceration of the os and cervix uteri, in which the mucous membrane of the cervical canal becomes oftentimes everted to a considerable extent, as well as hypertrophied and exceedingly vascular, a condition which increases the previously existing tendency to hæmorrhage. Thus, in not a few cases, we find these two causes present in the same patient. The following was quoted as an example of this:-

Mrs. F., the mother of twelve children, a woman aged forty-eight, presented herself a year previously at the dispensary of the Adelaide Hospital. She stated that ever since her last confinement, five years vol. IrI., NO. 104, N.s. 
ago, menstruation had gradually become more profuse, the flow continuing for a longer period than usual, the intervals between the periods being correspondingly shortened. During the intervals she suffered from profuse leucorrhea, and, as a result of this incessant drain, had become greatly debilitated. On examining the uterus the sound passed to the depth of three inches and a half. The os uteri was patulous; there was extensive ulceration of the os uteri; and on separating the lips, the mucous membrane lining the cervical canal was found to be thickened and highly vascular. This was a case requiring active treatment, which could not be adopted as long as the patient followed her ordinary occupation. She was accordingly admitted into hospital. Dr. Atthill at first proceeded to make an exploration of the uterine cavity, by dilating the cerfical canal and os internum. This was effected by the introduction of severul pieces of sea-tangle bougies. This enabled him to decide that there was not any polypus or fibrous tumour in the uterus. The inner surface of the uterus was then cauterized freely with the strong nitric acid, the application of which did not cause any pain. The patient was kept in bed for three or four days subsequently as a measure of precantion. On examining her after the lapse of a week, the condition of the ulceration which existed round the lips of the os was found to have improved considerably, and she was discharged in a short time perfectly cured.

In the foregoing case sub-involution was regarded as manifestly the primary cause of the menorrhagia, the ulceration being altogether secondary. In many cases, however, sub-involution exists alone, or, on the other hand ulceration may exist alone, either condition being fully sufficient to give origin to the menorrhagia. The following ease is an example of the former:-

F. L., aged twenty-four, married about a year, was a delicate young woman of lymphatic temperament. Menstruation had always been profuse, especially if she took walking exercise or exerted herself during the flow. She became pregnant after the occurrence of the second menstrual period following her marriage, but having imprudently taken a long and fatiguing walk, she aborted about the eighth week; two subsequent menstrual periods were so profuse as to reduce her to a state of extreme debility. Ergot, gallic acid, \&c., failed to do good. On examining her after the termination of these periods, the uterus proved to be considerably elongated, the sound passing to the depth of three inches and a half; there did not exist any ulceration. The history of the case being altogether against the supposition of the existence of polypus, Dr. Atthill came to the conclusion that the menorrhagia depended on subinvolution. In fact that the uterus had never regained its normal size and tone, since the miscarriage, which had occurred two months previously. He therefore determined to carry out a plan of treatment, the value of which he has repeatedly tested, namely, the introduction 
up to the fundus of the uterus of ten grains of the solid nitrate of silver, leaving it to dissolve there. This was accordingly done in this case. The application produced considerable pain, but no further unpleasant results followed. She was confined to bed for several days afterwards, but then allowed to go about. Menstruation appeared at the regular time and was moderate in quantity. This patient became pregnant immediately afterwards.

Dr. Atthill regards this case as a well marked illustration of the occurrence of sub-involution as a result of abortion--a fact which he considers is overlooked by many; next as showing the dangerous menorrhagia which may depend on this condition of the uterus; and, thirdly, as proving the excellent results which follows the treatment adopted. Ergot, gallic acid, and, indeed, all other medicines will fail to check menorrhagia depending on sub-involution; and we must have recourse to treatment directed to the uterus itself ; we must stimulate the organ to set up that healthy action by which it regains its normal size after pregnancy has terminated-a process to which Sir J. Simpson has applied the term "involution." With this view Dr. Atthill unhesitatingly advocates the adoption of the treatment practised in the preceding case, and asserts that be knows no other so efficacious. The mode of carrying it out is simple. The instrument known as Sir James Simpson's " uterine porte caustique" is introduced into the uterus just as an ordinary uterine sound.

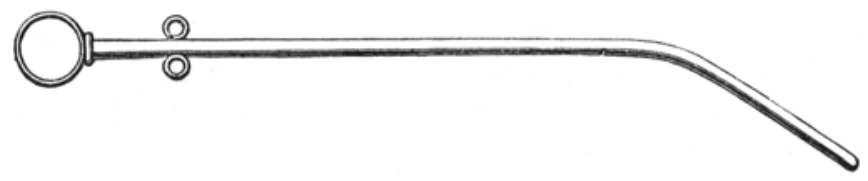

Uterine Porte Caustique.

This little instrument consists of a hollow silver tube, in size and shape closely resembling a sound. It contains a flexible stilette which it fits accurately. As soon as its point is found to have reached the fundus of the uterus, the stilette is withdrawn, and through the instrument is pushed up, by means of the stilette, a piece of solid nitrate of silver reduced to the requisite size and weight, till it is fairly lodged in the cavity of the uterus. The precaution must be taken, however, of withdrawing the instrument to the extent of half an ineh, as soon as the piece of nitrate of silver reaches the extremity of the porte caustique, and before it is finally pushed out of the instrument, a point of which we can always be certain by observing how much of the stilette remains still unintroduced; if this precaution be not observed, it is possible that the nitrate of silver might be forced into the substance of the uterine wall, instead of being left free in its cavity, an accident which, though possible, is very unlikely to occur. Dr. Atthill has practised this treatment for several 
years, and he believes that it is safe and simple. He does not say that it is always sufficient, and that a cure must always result; but in his hands it has been productive of marked success, and in no single instance has be known of its producing serious symptoms. The application of solid nitrate of silver to the interior of the uterus is by no means a novel practice. Dr. Evory Kennedy used to introduce it into the cavity of the body of the womb by means of an ordinary female catheter several years ago, but he did not leave it there to dissolve. To Sir J. Simpson we are indebted for the further development of the practice, and for the invention of the porte caustique. Dr. Atthill believes that Dr. Kidd was the first of the Irish obstetricians who adopted his practice.

Menorrhagia resulting from ulceration of the os and cervix uteri is also of frequent occurrence. Mere abrasion up the os uteri is not sufficient to produce menorrhagia; but that unhealthy spongy condition of the os and cervix, in which the mucous membrane lining its canal becomes hypertrophied and thickened, and bleeds on the slightest touch, the os being patulous and the lips everted, is quite capable of originating severe menorrhagia.

Mrs. B., a young married woman, aged twenty-four, who had never been pregnant, stated that she had become greatly debilitated by the excessive loss which occurred at each monthly period. Ergot and astringents were exhibited by the mouth, and astringent lotions injected into the vagina without producing the least effect. The use of the speculum proved the existence of exfensive granular ulceration of the os and cervix uteri. In such severe cases, the ulceration extends at least as high as the os internum, and we will fail to effect a cure unless our treatment reach every portion of the diseased tissue ; therefore with the view of permitting the necessary applications to be made to the whole of the extent of the cervical canal, in this case two tents of compressed seatangle were introduced, the object being to dilate the cervix to an extent sufficient to allow of his treating the entire extent of the cervical canal. He left these pieces of the sea-tangle in situ for twenty-four hours, and on withdrawing them after the lapse of that time, cauterized freely the whole diseased surface with fuming nitric acid. This did not cause any pain. On examining the os uteri a few days subsequently be found it in a much healthier state. The menorrhagia was entirely checked and never returned; and although a considerable time elapsed before the uterus regained a healthy state, still the progress of the cure was rapid and the cure perfect; the only treatment subsequently necessary being the occasional application of a twenty grain solution of nitrate of silver to the os uteri, and at a later period, of a small blister to the sacrum ; finally, not the slightest trace remained of the ulceration, and menstruation became in all respects normal. 
Dr. Atthill states that in cases of granular ulceration of the os and cervix uteri, of course it is not always necessary to dilate the cervix uteri If the case be recent, and if we can satisfy ourselves that the ulceration does not extend very high, the use of the solid nitrate of silver, of zinc points, or brushing the parts lightly over with nitric acid, may be sufficient, but in the graver forms of the disease the only effectual treatment will be that just advocated. Dr. Atthill believes that not a little of the opprobrium which rests on obstetric practitioners from the length of time over which this treatment extends is due to excessive timidity, and to the use of inefficient remedies. The case above related was an example of so-called granular ulceration of the mucous membrane of the os and interior of the cervix ateri. Properly speaking, however, there is no true ulceration in this case. On the contrary, the mucous membrane is thickened and hypertrophied, its surface being covered with numerous papillæ, which, from the resemblance they present to ordinary healthy granulations, have given origin to the term "granular ulceration." These are not new growths, however, being merely enlargements of the ordinary papillæ which have, under the influence of disease, become enlarged and vascular, and bleed on the slightest touch. This condition is, by most writers, spoken of as never extending beyond the os internum; it does not do so generally, but Dr. Atthill has no doubt whatever that the same condition which we see in the cervical canal, also not unfrequently exists within the cavity of the uterus, and is the result of causes similar to those which originated it in the former situation. If we meet with a case of menorrhagia in an otherwise healthy woman, which a careful vaginal examination proves not to depend on granular ulceration of the os or cervix, or on sub-involution, it is our manifest duty, continues the author, to dilate the cervix and os internum, with the view of determining what the condition of the interior of the body of the organ may be. As a rule the uterus is seldom in such cases much elongated, the increase being not more than to the extent of perhaps half an inch. This point is of importance in enabling us to decide as to the possible presence of an intra-uterine tumour; but the existence of these or of the condition under consideration can only be solved by dilating the cervix, and then passing the finger fairly up to the fundus of the uterus. It is surprising how little the patient suffers from this process, and how rapidly the os regains its natural size. Not less remarkable is the entire absence of all unpleasant symptoms after a proceeding apparently so severe; and he has not the least hesitation in recommending the practice.

For dilating the cervix he has quite given up the use of sponge tents, and invariably adopts the plan recommended by Dr. Kidd of introducing a number of pieces of sea-tangle bougies. If the cervix be rigid three or four pieces will be as many as can be safely inserted ; but if it be relaxed, double, or even treble, that number may be with impunity inserted. If 
the smaller number be used they should be withdrawn after the lapse of a few hours, and a larger number inserted; but in my case the os internum must be dilated to a size sufficient to admit the top of the index finger to pass through. To effect this by no means easy matter, the first step after withdrawing the sea-tangle is to seize the anterior lip with a vulsellum, and with it to draw the uterus well down. This should be done by an assistant; pressure being at the same time made on the fundus; by these means the uterus will have been brought so low, that unless the pelvis be very deep the point of the finger will reach the very fundus, and we are enabled to discover the presence of even a very small polypus, should it exist, or to detect the rough uneven feel of a granular condition of the mucous membrane, which Dr. Atthill regards as a frequent cause of menorrhagia. 'This condition he considers the result of subacute inflammation or congestion of the mucous membrane. In order to effect a cure it is necessary to destroy the so-called granulation, and to endeavour to excite a healthy action in the diseased part. With this view, he employs the strong nitric acid, which he applies freely to the entire of the inner surface of the uterus, by means of a bit of lint fastened to a proper holder. In this proceeding the os should be brought into view by the duck-bill speculum, and while the anterior lip is drawn down by the vulsellum he passes the stick armed with the lint dipped in nitric acid through the os internum, and sweeps it freely but rapidly round the interior of the uterus. Another piece of lint soaked in water is then passed up to the os, to proteet the vagina from any acid discharge from the os uteri, and the vulsellum and speculum being withdrawn, the patient is left in bed for some days, absolute rest being required. With due attention to these precautions no ill effects need be dreaded. The communication concluded with the details of cases treated in this manner.

Menorrhagia of Six Year's Duration, depending on an Intramural Tumour of the Anterior Wall of Uterus, treated by the application of strong Nitric Acid-Recovery. By Henry GogarTy, F.R.C.S.I.-On the 7th of April, 1870 , I was sent for to see Mrs. E., who was reported to be suffering from a considerable loss of blood. On my arrival at her home, $I$ found her almost pulseless, perfectly anæmic, nearly unable to articulate, and deluged in blood. She complained of noise in the ears, giddiness, and swimming in her head, and dimness of vision.

My first impression was that she had aborted, and that some of the secundines remained in the cervix or os uteri, giving rise to the profuse hæmorrhage. However, subsequent inquiry and examination convinced me that such was not the case.

She being unable, from exhaustion, to give me any further account of herself, I directed my efforts to arrest the bleeding and restore her 
strength. This I succeeded in accomplishing, in a few days, by the administration of stimulants, nutrition, gallic acid and opium, with small doses of liquor extract of ergot in glycerine (a preparation introduced by Dr. Long, of the firm of Hamilton, Long, \& Co., Sackville-street, and which I have found to be more certain in its effects, and much less likely to nauseate than the ordinary extractum ergotæ liquidii of the pharmacopœia). Her history is as follows:-

Mrs. E., age $4 z$, always enjoyed good health up to six years ago. She married at 35, and conceived six months later. She went on in good health until the middle of the sixth month of pregnancy, when, without any apparent cause she aborted, and made but a poor recovery. From this time she dates her ill health. She noticed that the catamenia came regularly, but remained with her longer than usual. A few months later, the quantity increased. Still, her general health did not suffer much. Soon afterwards, the flow became more profuse; but she attributed this to the circumstance of her husband having returned from the Mediterranean and living constantly with her. Two months after his return, she found her strength beginning to fail, complained of weakness, amounting to faintness, lassitude, and want of energy. At length she consulted a physician, who prescribed astringents, in various forms, together with rest in the recumbent position, nourishment, \&c. This treatment was continued, with varying degrees of success, for the space of four years, the last three of which she was scarcely ever free of bloody discharge.

She now resolved to give up doctors, as she benefited nothing by medicine, and was informed by a midwife that, probably, her change of life was at hand, and all would be well when menstruation ceased. The unction of this old woman's comforting words was not destined to remain long with her, for the arrival of an American lady (a relative of her husband, furnished with speculum, continuous vaginal syringe, and sundry "pacquets" of F'rench simple astringent powders for injecting the vagina) blasted the soothing influence instilled into her mind by the elderiy female, and cast her again on the troubled waters of doubt and fear.

Soon the paraphernalia were got in readiness, and many a quart of fluid was injected into the vagina, but to no purpose, for still the blood it came. A week after the departure of this modern matron of the new world, I first saw her, in the condition before depicted.

A fortnight after my first visit her strength had so much improved that I made up my mind to explore the uterus, and ascertain its exact dimensions, and the extent of its cavity. I placed her in the simiprone position, recommended by Dr. Marion Sims, and with his duckbill speculum, and the assistance of an intelligent woman, I found the os externum healthy, and slightly opened, a small stream of blood flowing from it. The cervix was considerably elongated, the cavity measuring 
five and a half inches. When the sound reached the os internum, I experienced some difficulty in getting it beyond that point, and had to resort to various inclinations of its axis to overcome this barrier. She was next put in the lithotomy position, and with the greatest facility, by the bi-manual manipulation, I ascertained that the body was enlarged, the anterior wall was more convex and thickened than the normal uterus.

Having thus satisfied myself of the enlargement, I suspected the existence of a tumour, and felt that it was necessary to dilate the cavity, in order to learn if such existed, its nature, and to apply remedial measures, if expedient. This I accomplished by the gradual introduction of sea-tangle tents. On the fifth day I had inserted seven tents of the size of No. 6 bougie. When those were fully expanded by the evening of the same day, the uterus was fairly open, and admitted freely two fingers. On examining the interior, $I$ discovered a medium-sized intramural tumour, situated in the anterior wall, reaching from the os internum to the fundus.

The information now obtained perplexed me not a little, for I was at a loss to know what to do, under the circumstance.

I returned five of the tents, together with a fresh one, and resolved to wait the morrow, to deliberate on what was best and safest to be done. The mucous nembrane covering the tumour, and, indeed, all the mucous lining of the uterus, was intensely vascular, the slightest exertion or excitement causing it to bleed freely. To let my patient drain to death was a serious reflection; but what could $I$ do? The idea of gouging the tumour, as recommended by Mr. Baker Brown, presented itself to my mind; but, as I had no experience of it, I did not like to hazard her life by so formidable an operation.

The next idea which struck me was the injection of the uterine cavity with compound tincture of iodine, as practised by Dr. Savage. This proceeding I feared, for I have considerable experience of the serious inflammation it sets up when employed as an injection into the tunica vaginalis, for the radical cure of hydrocele.

A short time before this case came under my care, I read in one of the journals an article on the application of strong nitric acid to the internal surface of the uterus, for the removal of sessile growth from its wall. I had witnessed its beneficial effects in the practices of my friends Ioctors Kidd and Roe, at the Coombe Hospital. Thus encouraged by experience, and fortified by the knowledge that if it did not succeed, at least it would do no harm, I resolved next day to apply it freely to the tumour in hope it might shrink it, or arrest its development towards the uterine cavity. With the aid of Mr. Wyer, who examined the case, and kindly assisted me in taking charge of the speculum and vulcellum, having previously anointed the vagina with lard, I mopped the tumour freely and over its whole extent with the acid, had her put to bed, and ordered 
a full opiate. Next morning, she stated she had slept well, had had no pain or bleeding, and felt very comfortable. She remained without change for three days. On the fourth day. a slight sanious discharge appeared; yet there was no pain or other disturbance. On the fifth day, the discharge increased with slight fœetor. She was ordered to be syringed with a weak tepid solution of Condy's permanganate of potass. Sixth day, discharge more abundant and more fotid; had a good night, pulse better, and desired food. I placed a tampon of cotton, saturated with glycerine, in the vagina, and ordered ten grains of chlorate of potass with bark, to be taken every third hour. The glycerine increased the discharge very much, but quite removed the smell. On the eighth day, she complained of stinging pains in the lower part of abdomen. The discharge, about the same in quantity, became redder. I examined her with the speculum, and found protruding from the os a membrane which I cautiously withdrew with a pile forceps. This proved to be a complete caste of the uterine face of the tumour, formed of the mucous lining of the uterus, which was denuded by the acid. From this date the discharge gradually diminished, and left her perfectly free on the thirteenth day after the operation. Sixteen days later, she menstruated, the catamenia lasting four days, being healthy in quantity and quality. She was removed to Dalkey for change of air, and ordered iron and quinine. She has never had a bad symptom since: has gained three stone in weight, is stout and florid, and can attend, with ease and romfort, to her household duties.

I saw her in March of this year. She was then well, strong, and cheerful.

I thought, Sir, that the details of this case would not be uninteresting to the members of this Society, the more so on account of the interest evinced in the etiology, pathology, and treatment of uterine fibroids by nearly all the leading gynæcologists in Great Britain and America at the present time. I am also desirous to elicit the opinions of our members as to their experience of medicinal agents given by the mouth, and acting through the general circulation, on those growths, as much has been said, both for and against, by the highest authorities here and in the sister countries.-May 13, 1871. 